\section{GROUP-A BETA-HEMOLYTIC STREPTOCOCCI RESISTANCE PATTERN IN NORTH INDIAN PEDIATRIC POPULATION}

Sir,

The interesting article by Jain et al. describes the resistance pattern of GABHS (group-A beta-hemolytic streptococci) in northern India..$^{[1]}$ The article may be of immense interest to all clinicians. The authors mention in the abstract section that the current resistance pattern of GABHS has not been discussed in India, which is incorrect. A thorough search of literature reveals that Lloyd et al. had conducted a study on 117 strains of group $A$ streptococcus (GAS), 60 strains of group $G$ streptococcus (GGS) and 20 strains of group C streptococcus (GCS). ${ }^{[2]}$

The authors have not mentioned, anywhere in the article, the exact place of the study. Northern India is a vast region, and it is necessary to specify a particular region. It is also sure that there are many cosmopolitan cities in northern India which have a mixed population, and the results are bound to vary. Information regarding the exact socioeconomic status of the subjects in the pediatric age group would have thrown more light on the matter.

A closer look at Table 1 indicates that both the sample size and the positivity towards GABHS decreased with an increase in age. A better statistical test would have spelt out the significance of the results. The present study lacks proper statistical tests, and it simply highlights the frequency of the positive culture without comparing any significance between the groups.

The authors should have explained the clinical reasons for the increase in resistance pattern of tetracycline and erythromycin and the sensitiveness towards penicillin. Obviously, genetic factors might have also played an important role. All the factors of drug resistance are to be considered for the interpretation of results. It is important to know the mutations in order to label a macrolide as a resistant one. Also, a complete history of the adverse drug reactions would have helped clinicians to try the best drug under such circumstances.

Earlier studies had even looked into the molecular heterogeneity among the clinical isolates of group-A streptococcus in the north Indian population, which might even help in vaccination. ${ }^{[3]}$ While comparing the results of this study with those of studies in other countries, the geographical location which plays an important role has to be kept in mind. Overall, it is an interesting article and might be beneficial to all clinicians, and the editor deserves special applause for publishing articles on such important topics. 
SRIJIT DAS

Department of Anatomy, Faculty of Medicine, Universiti Kebangsaan Malaysia, Jalan Raja Muda Abd Aziz, 50300 Kuala Lumpur, Malaysia

Correspondence: E-mail: das_srijit23@rediffmail.com

\section{References}

1. Jain A, Shukla VK, Tiwari V, Kumar R. Antibiotic resistance pattern of group: A beta-hemolytic streptococci isolated from north Indian children.
Indian J Med Sci 2008;62:392-6.

2. Lloyd CA, Jacob SE, Menon T. Pharyngeal carriage of group A streptococci in school children in Chennai. Indian J Med Res 2006;124:195-8.

3. Sagar V, Bakshi DK, Nandi S, Ganguly NK, Kumar $\mathrm{R}$, Chakraborti A. Molecular heterogeneity among north Indian isolates of group A streptococcus. Lett Appl Microbiol 2004;39:84-8.

DOI: DOI:10.4103/0019-5359.56112

Announcement

Dr. J . C. Patel Medical Research Foundation is organizing Fifth Conference on Iron Deficiency at Shanti Sarovar, Hyderaban on 5 -7 February 2010. Early registration closes on 31/8/09. Only first 150 out-station delegates w ould be registered and would be provided free accommodation. Local delegates not requiring accommodation will have unrestricted registration. Last date for submission of abstracts for free papers is $15 / 11 / 09$. All accepted abstracts w ould receive cash awards based on merits.

For details conact: For outstation delegates - Dr. B. C. M ehta, 504, Pracho Society, J uhu-Versova Link Rd, Andheri (W) M umbai 400053.

E-mail iconid2008@gmail.com Web-sitewww.ghrc.bk.org 